\title{
Ginástica Laboral: efeitos de um programa de ginástica laboral sobre a flexibilidade em trabalhadores de diversos setores de um hospital de ensino da região do Vale do Rio Pardo/RS
}

\author{
Effects of a Labor Gymnastics program on flexibility of workers in \\ various sectors of a teaching hospital from vale do rio pardo region
}

Andréa Mattos de Andrade', Cézane Priscila Reuter', Miriam Beatris Reckziegel'1, Hildegard Hedwig Pohl', Leandro Tibiriçá Burgos'

Universidade de Santa Cruz do Sul (UNISC), RS, Brasil.

Recebido em: outubro 2015 / Aceito em: novembro 2015

Iburgos@unisc.br

\section{RESUMO}

A Ginástica Laboral (GL) é uma atividade física realizada durante a jornada de trabalho, com exercício de compensação aos movimentos repetitivos, à ausência de movimentos, ou a posturas desconfortáveis assumidas durante o período de trabalho. Objetivo: verificar possíveis efeitos de um programa de GL sobre a flexibilidade em trabalhadores de diversos setores de um hospital de ensino da região do Vale do Rio Pardo/RS. Método: estudo semi-experimental realizado em diversos setores de um hospital, de março de 2014 a maio 2015, em que foram avaliados 42 indivíduos, do sexo feminino, com idade entre 22 e 58 anos, que participavam do programa de GL. Para a coleta de dados, foi aplicado o teste sentar e alcançar (sit and reach) e o teste do manguito rotador (ombro direito e esquerdo) em três momentos: 1 (antes da intervenção com GL); 2 (logo após o término da intervenção) e 3 (quatro meses, após a intervenção). Resultados: a flexibilidade diferiu na comparação entre os momentos 1 e 2 e entre os momentos 1 e 3, em todos os testes aplicados, demonstrando que o programa de GL melhorou os níveis de flexibilidade dos trabalhadores. Não foi observada diferença significativa entre os momentos 2 e 3. Considerações finais: o programa de GL melhorou os níveis de flexibilidade dos trabalhadores, mantendo esses níveis semelhantes seis meses após o término da intervenção.

Palavras-chave: Educação Física; Ginástica laboral; Flexibilidade.

\section{ABSTRACT}

The labor gymnastics (LG) is a physical activity performed during the workday, with clearing exercise to repetitive movements, absence of movement, or uncomfortable positions taken during the working period. Objective: to investigate the effects of a $L G$ program on flexibility of workers in different sectors of a teaching hospital from Vale do Rio Pardo region - RS. Method: semi-experimental study carried out in various sectors of the Hospital Santa Cruz from March 2014 to May 2015 The stydy evaluated 42 individuals, female, aged between 22 and 58 years, who participated in the LG program. To collect data the Sit and Reach test and the rotator cuff test were applied. Results: the three applied flexibility tests showed significant differences ( $p<0.001)$ when compared. There was an increase with respect to flexibility, indicating the positive perception of $L G$ effects. Final considerations: $L G$ program improved flexibility levels of workers. There was no significant difference between times 2 and 3 observed for the three tests. Thus, subjects maintained similar levels of flexibility even 6 months after the end of the intervention. Flexibility.
Keywords: Physical Education; Labor Gymnastics; 


\section{INTRODUÇÃO}

O estilo de vida da população modificou-se nas últimas décadas, gerando uma elevação do número de pessoas que se tornaram sedentárias, especialmente em países industrializados. ${ }^{1}$ Por outro lado, observa-se uma redução das oportunidades de praticar atividades físicas, o que está relacionado, tanto à morbidade, quanto à mortalidade. As doenças infecciosas foram substituídas por um enorme avanço das enfermidades crônicas e daquelas originadas pelo homem. ${ }^{2}$ Pesquisas têm mostrado associação entre os baixos níveis de atividade física e o desenvolvimento de doenças cardiovasculares, câncer, diabetes e agravos na saúde mental, ${ }^{1-3}$ com a consequente redução da qualidade de vida da população, ${ }^{4}$ fatores que comprometem também os trabalhadores.

Devido à globalização e a intensificação da pressão organizacional, tem aumentado à jornada de trabalho e nível de exigências dos trabalhadores, podendo comprometer a saúde física e mental dos mesmos. ${ }^{5} \mathrm{Um}$ trabaIho que demanda a realização de tarefas extremamente fragmentadas, altos níveis de concentração e atenção associados a diversas normas de produção, repercute de forma negativa sobre a saúde psíquica do indivíduo, podendo ocasionar o estresse ocupacional. $O$ estresse ocupacional é um fenômeno que está relacionado ao indivíduo e seu ambiente de trabalho. As demandas ocupacionais quando percebidas como pressão podem descompensar o equilíbrio do corpo, gerando respostas adaptativas (comportamentais, afetivas, cognitivas e fisiológicas) chamadas de estresse. ${ }^{6}$

A carga de trabalho, o esgotamento, os conflitos, a sobrecarga de função e a falta de controle sobre o próprio trabalho, são julgados fatores que tornam o trabalho estressante. Assim, torna-se importante agir na prevenção do aparecimento de agentes estressores, sendo que o estresse relacionado ao trabalho pode ser um dos mais evitáveis problemas de saúde. Neste sentido, é possível identificar quatro fases distintas do estresse: a primeira fase conhecida como alarme ou alerta, a segunda como resistência, a terceira como quase exaustão e quarta como exaustão. ${ }^{7}$

$\mathrm{Na}$ atualidade, as organizações diante das demandas do mercado, ao mesmo tempo em que expõem os indivíduos a níveis elevados de tensão e estresse, têm voltado suas discussões para programas de qualidade de vida e prevenção de doenças. Essas discussões tornam-se ainda mais evidentes e necessárias, à medida que se observa a influência positiva e os diversos benefícios no investimento na saúde e qualidade de vida do profissional (funcionário). Estas questões devem seguir paralelas aos objetivos de crescimento da empresa e organizações, garantindo, consequentemente, qualidade na prestação de serviços e produtos, bem como no processo de produção e execução de serviços. ${ }^{8}$

A realidade do serviço hospitalar não é diferente de outros serviços, gerando sobrecarga e cobrança por produtividade como fator estressante para seus funcionários, com um ritmo exaustivo de trabalho, inclusive com regime de plantões, carga horária elevada, turno noturno, trabalhadores com mais de um emprego devido aos baixos salários, enormes responsabilidades, risco de acidentes, atividades antiergonômicas e atividade física insuficiente. Assim, ao fim do expediente, muitos desses trabalhadores encontram-se com algias musculares, nas articulações e nos membros, em consequência de posturas inadequadas durante a realização das atividades relacionadas a cada profissão. ${ }^{9}$

Os programas de saúde ocupacional que visam à eliminação ou a redução dos riscos a que os funcionários estão expostos em seu ambiente de trabalho, com atitudes especialmente proativas, ou seja, aquelas que se antecedem às situações de risco de acidentes, doenças ocupacionais, danos materiais ou ambientais, visando à minimização de custos e melhoria das condições de trabalho dos trabalhadores, são cada vez mais importantes. ${ }^{10}$

Neste contexto, surge a GL, definida como uma atividade física praticada no local de trabalho de forma voluntária e coletiva pelos funcionários na hora do expediente, como uma ferramenta disponível dentro da ergonomia desenvolvida em uma empresa, no sentido de prevenir as doenças ocupacionais, contribuindo para melhoria da qualidade de vida dos funcionários. Hoje, produzir mais e melhor, sem desenvolver danos à saúde dos trabalhadores é uma necessidade, não se tratando de uma utopia. ${ }^{11-13}$

Torna-se indispensável o desenvolvimento desse estudo, pois se trata de uma realidade distante da maioria das pessoas que trabalham de modo ininterrupto e extenuante, decorrente do número insuficiente de funcionários para atender uma demanda crescente, levando atualmente a preocupação de algumas instituições com o bem-estar do corpo de funcionários, do que decorre um atendimento mais qualificado para atender às necessidades dos pacientes. Julgamos viável o desenvolvimento desse estudo, uma vez que os trabalhadores da área da saúde encontram-se sujeitos a inúmeros riscos no trabalho sem receberem dos serviços em que operam apoio aos danos gerados pelo regime de trabalho, diminuindo a capacidade laboral e a qualidade do trabalho ofertado. ${ }^{9}$

O presente estudo tem como objetivo verificar quais os possíveis efeitos de um programa de ginástica laboral sobre a flexibilidade em trabalhadores de diversos setores de um hospital de ensino da região do Vale do Rio Pardo/RS.

\section{MÉTODO}

Os sujeitos do presente estudo semi-experimental são 42 trabalhadores em atividade laboral regular, sendo toda a amostra do sexo feminino, funcionárias de um hospital de ensino da região do Vale do Rio Pardo/RS.

Para a coleta de dados foram utilizados o teste Sentar e alcançar (Sit and Reach) (ACSM, 2000) - TSA o qual, é um teste ativo, que mede a flexibilidade da articulação do quadril e avalia os músculos paravertebrais e os posteriores da coxa, bem como o teste deExtensão de ombros - Teste do manguito rotador. ${ }^{13}$ Todos os procedimentos de avaliação foram realizados em uma única sessão.

O treinamento dos indivíduos foi realizado de forma sistematizada, prescrito em microciclos, mesociclos e macrociclos. ${ }^{14}$ As intervenções do programa de GL foram realizadas durante seis meses, três vezes por 
semana, aproximadamente quinze minutos; às sessões são estruturadas com exercícios funcionais, resistência muscular localizada, exercícios de alongamento e relaxamento, com um período de 04 meses de intervalo para o reinício do programa.

A análise dos dados foi realizada no programa SPSS v. 20.0 (IBM, Armonk, Ny, USA), através do teste não-paramétrico de Friedman. A comparação dos testes avaliados, nos três períodos (pré-teste, pós-teste e quatro meses após a intervenção), foi realizada por meio do teste Post-hoc de Dunn-Bonferroni, considerando significativas as diferenças para $p \leq 0,05$.

O estudo foi aprovado pelo Comitê de Ética em Pesquisa da Universidade de Santa Cruz do Sul (UNISC). Todos os indivíduos que concordaram em participar assinaram um termo de consentimento livre e esclarecido.

\section{RESULTADOS}

São participantes do programa aproximadamente 150 funcionários, mas integraram esta pesquisa 42 indivíduos, do sexo feminino, com idade entre 22 e 58 anos, em atividade laboral regular, sendo excluída uma trabalhadora devido à gestação, duas trabalhadoras desligadas da instituição no decorrer do trabalho de pesquisa e cinco trabalhadoras tiveram alterações em seus turnos de trabalho.

Os três testes de flexibilidade aplicados apresentaram diferença significativa $(p<0,001)$ na comparação dos três momentos. Em todos os testes, observa-se um aumento na flexibilidade do momento 1 (antes da intervenção com GL) para o momento 2 (logo após a intervenção). Os resultados indicaram a percepção positiva dos efeitos da GL com relação à flexibilidade.

$\mathrm{Na}$ avaliação de quais momentos os testes de flexibilidade diferem significativamente (Tabela 2), observa-se que, para todos os testes, a flexibilidade difere na comparação entre os momentos 1 e 2 e entre os momentos 1 e 3 , demonstrando que o programa de GL melhorou os níveis de flexibilidade dos trabalhadores. Não foi observada diferença significativa entre os momentos 2 e 3, para os três testes avaliados; dessa forma, os sujeitos mantiveram os níveis de flexibilidade semelhantes, 6 meses após o término da intervenção.

\section{DISCUSSÃO}

Os três testes de flexibilidade aplicados apresentaram diferença significativa $(p<0,001)$ na comparação dos três momentos. Em todos os testes, observa-se um aumento na flexibilidade do momento 1 (antes da intervenção com GL) para o momento 2 (logo após a intervenção). Os resultados indicaram a percepção positiva dos efeitos da GL com relação à flexibilidade.
Tabela 2 - Comparação dos testes de flexibilidade nos três momentos.

\begin{tabular}{lc}
\hline Teste & $\boldsymbol{P}^{*}$ \\
\hline Sentar e alcançar & \\
Momento 1 - Momento 2 & $<0,001$ \\
Momento 1 - Momento 3 & $<0,001$ \\
Momento 2 - Momento 3 & 0,057 \\
Manguito rotador - OD & \\
Momento 1 - Momento 2 & $<0,001$ \\
Momento 1 - Momento 3 & $<0,001$ \\
Momento 2 - Momento 3 & 1,000 \\
Manguito rotador - OE & \\
Momento 1 - Momento 2 & 0,005 \\
Momento 1 - Momento 3 & $<0,001$ \\
Momento 2 - Momento 3 & 0,900 \\
\hline
\end{tabular}

Momento 1: pré-teste; momento 2: pós-teste, após a intervenção com GL; momento 3: 6 meses após o término da GL; *teste Post-hoc de Dunn-Bonferroni.

Observa-se que, para todos os testes, a flexibilidade difere na comparação entre os momentos 1 e 2 e entre os momentos 1 e 3 , demonstrando que o programa de GL melhorou os níveis de flexibilidade dos trabalhadores. Não foi observada diferença significativa entre os momentos 2 e 3 , para os três testes avaliados; dessa forma, os sujeitos mantiveram os níveis de flexibilidade semelhantes, 6 meses após o término da intervenção. Os resultados do presente estudo corroboram evidências anteriormente apresentadas em estudos sobre a flexibilidade.

Estudo realizado na Espanha, ${ }^{15}$ com 58 mulheres trabalhadoras de uma indústria $(\mathrm{GE}=27 ; \mathrm{GC}=31)$, com o objetivo de verificar o efeito de um programa de alongamento realizado no local de trabalho, sobre a extensibilidade dos músculos isquiotibiais e postura sagital da coluna vertebral, verificou, no grupo experimental, aumento significativo da inclinação pélvica no teste de envergadura e diminuição significativa na curva torácica. Constataram, portanto, que exercícios de alongamento realizados no local de trabalho, são eficazes para aumentar a extensibilidade dos músculos isquiotibiais.

Um estudo ${ }^{16}$ realizado com 10 funcionários da Secretaria Municipal de Educação de Taquari (RS), verificou a flexibilidade do quadril e a flexibilidade dos ombros. Para apresentação dos resultados, os sujeitos foram divididos em dois grupos, um do sexo feminino e outro do sexo masculino; foi observado que a maioria dos sujeitos aumentou a amplitude de movimento; além disso, notou-se que as mulheres tiveram um aumento maior que os homens, sendo que a média de ganho de flexibilidade feminino foi de $1,57 \mathrm{~cm}$ e masculino 1,0 $\mathrm{cm}$. Este resultado vai ao encontro dos argumentos que afirmam que no sexo feminino é percebida maior flexibilidade que o sexo masculino em todas as idades; talvez pelas atividades que exigem maior uso de flexibilidade

Tabela 1 - Comparação dos testes de flexibilidade nos três momentos.

\begin{tabular}{lcccc}
\hline WHQ & Momento 1 & Momento 2 & Momento 3 & $\boldsymbol{P}^{*}$ \\
\hline Sentar e alcançar $(\mathrm{cm})$ & $24,00(9,51)$ & $29,57(9,30)$ & $28,05(9,11)$ & $2,59(7,09)$ \\
Manguito rotador - OD & $0,05(6,62)$ & $3,17(7,37)$ & $-0,001$ & $<, 29(8,51)$
\end{tabular}

Momento 1: pré-teste; momento 2: pós-teste, após a intervenção com GL; momento 3: 6 meses após o término da GL; OD: ombro direito; OD: ombro esquerdo; ${ }^{*}$ teste de Friedman. 
do sexo feminino e pelas atividades de força predominante do sexo masculino. ${ }^{17}$

$\mathrm{Na}$ pesquisa de Zatarin e Bortolazzo ${ }^{18}$ realizada na Clínica de Fisioterapia da Faculdade Anhanguera de Piracicaba - SP, com o objetivo de avaliar os efeitos da manipulação na articulação sacro-ilíaca e transição lombossacral sobre a flexibilidade da cadeia muscular posterior de 21 mulheres com idade entre 18 e 40 anos (média de 26,2 $\pm 6,1$ anos), sem incapacidade funcional por lombalgia assintomáticas, alocadas em dois grupos, controle (GC, $n=10$ ) e experimental (GE, $n=11)$. Ambos os grupos foram avaliados (pré e pós-intervenção imediata) com os testes de Schober e de elevação do membro inferior estendido (TEMIE) (bilateralmente) e mensuração da flexibilidade da cadeia muscular posterior pelo Banco de Wells. O GE recebeu a manobra global na pelve (bilateralmente) como intervenção, enquanto que no GC elas apenas permaneceram em decúbito dorsal por 1 minuto. $\mathrm{Na}$ análise dos resultados intergrupos (pré-intervenção), não houve diferença significativa para nenhuma das variáveis analisadas. $\mathrm{Na}$ análise intergrupo (pré e pós) dos resultados do GC foi encontrada diferença significativa apenas para o Banco de Wells ( $p=0,0087)$; já no GE houve aumento significativo para o Banco de Wells $(p<0,0001)$ e para o TEMIE $(p=0,0488$ e $p=0,0172)$. Em relação aos resultados intergrupos (pós-intervenção), houve diferença significativa entre o GE e o GC $(p=0,0025)$ para o Banco de Wells, constatando-se que a manipulação na articulação sacro-ilíaca e transição lombossacral promoveu aumento da flexibilidade da cadeia muscular posterior em indivíduos assintomáticos do sexo feminino.

Em outra pesquisa ${ }^{19}$ realizada em Montes Claros - MG, com 23 indivíduos, sexo masculino, sendo 13 praticantes do $P G L$ (idade $=45,46 \pm 9,64$ anos, massa corporal $=75,88 \pm 10,17 \mathrm{~kg}$, estatura $=1,70 \pm 0,08$ $\mathrm{cm}, I M C=26,33 \pm 2,88 \mathrm{~kg} / \mathrm{m} 2)$ e 10 não praticantes do PGL (idade $=42,90 \pm 8,88$ anos, massa corpo$\mathrm{ral}=77,97 \pm 12,84 \mathrm{~kg}$, estatura $=1,71 \pm 0,05 \mathrm{~cm}, \mathrm{IMC}=$ $26,80 \pm 4,29 \mathrm{~kg} / \mathrm{m} 2$ ), para avaliar o nível de flexibilidade e qualidade de vida em praticantes e não-praticantes do programa de ginástica laboral em uma empresa de saneamento básico, submetidos aos testes de flexibilidade (goniometria do quadril, apley e schober), bem como a avaliação de peso e estatura foi constado que o grupo que participa do PGL obteve médias melhores na flexibilidade do quadril e ombro direito, comparado ao grupo que não participa, sendo: $F Q D=97,95 \pm 13,4$, $\mathrm{FQE}=96,15 \pm 6,45, \mathrm{FOD}=-12,46 \pm 11,69$. Em relação à flexibilidade da coluna lombar, o grupo que não participa da GL obteve média de 4,82\% maior que a média do grupo que participa da GL, porém, esta diferença não foi estatisticamente significativa, o que aconteceu também na flexibilidade da articulação do ombro esquerdo. Os resultados da pesquisa apontam que o nível de flexibilidade do GPGL é consideravelmente melhor que o nível do GNGL.

Os dados propõem a percepção positiva dos efeitos da GL pelos trabalhadores, identificando que esta parece ter trazido ampla contribuição, não apenas no que se refere aos aspectos avaliados nesta pesquisa, flexibilidade, mas também psicossociais e relacionais.

\section{CONSIDERAÇÕES FINAIS}

Concluiu-se que os testes de flexibilidade aplicados apresentaram diferença significativa, demonstrando que o programa de GL melhorou os níveis de flexibilidade dos trabalhadores. Não foi observada diferença significativa entre os momentos 2 e 3 , para os três testes avaliados; dessa forma, os sujeitos mantiveram os níveis de flexibilidade semelhantes 6 meses após o término da intervenção.

Recomenda-se que devem ser ampliadas as oportunidades de GL bem como as estratégias de saúde do trabalhador devem ser mais amplas do que somente a aplicação da GL, e esta deve ser parte integrante de uma política mais abrangente na valorização da saúde dos trabalhadores, devendo-se considerar que, juntamente com a GL, deve- se implementar um conjunto de melhorias, como modificação do processo de trabalho, instituição de revezamentos ou rodízios, realização de análises ergonômicas dos postos de trabalho e adequação dos instrumentos ou equipamentos de trabalho

\section{REFERÊNCIAS}

1. Pitanga FJ, Lessa I. Prevalence and variables associated with leisure-time sedentary lifestyle in adults. Cadernos de Saúde Pública 2005; 21(3): 870-887.

2. Murray CJL, Lopez AD. Mortality by cause for eight regions of the world: global burden of disease study. The Lancet 1997; 349(9061): 1269-1276.

3. Monteiro WD. Aspectos fisiológicos e metodológicos do condicionamento físico na promoção da saúde. Revista Brasileira de Atividade Física e Saúde, 1996.

4. Fontes ACD, Vianna RPT. Prevalência e fatores associados ao baixo nível de atividade física entre estudantes universitários de uma universidade pública da região Nordeste - Brasil. Revista Brasileira de Epidemiologia, São Paulo 2009; 12(1): 20-29.

5. Mendes AR, Leite N. Ginástica laboral: princípios e aplicações práticas. São Paulo, 2002; Manole. 2004; 2-1. Ação multiprofissional. 2 ed.

6. Ladeira MB. O processo de stress ocupacional e a psicopatologia do trabalho. Revista Administração, São Paulo 1996; 31(1): 64-74.

7. Straub RO. Psicologia da saúde: uma abordagem biopsicossocial. Porto Alegre: Artmed, 2005.

8. Cañete I. Humanização desafio da empresa moderna. São Paulo: Foco, 1996.

9. Gondim KM et al. Avaliação da prática de ginástica laboral pelos funcionários de um hospital público. Revista Rene 2009; 10(2): 95-102.

10. Lima V. Ginástica Laboral: atividade física no ambiente de trabalho. 3. ed. Revista Ampliada. São Paulo: Phorte, 2007.

11. Martins CO. Repercussão de um programa de ginástica laboral na qualidade de vida de trabalhadores de escritório. Tese (Departamento de Tecnologia) - Universidade Federal de Santa Catarina, Florianópolis (SC), 2005.

12. Martins GC, Barreto SMG. Vivências de ginástica laboral e melhoria da qualidade de vida do trabalhador: resultados apresentados por funcionários administrativos do instituto de física da Universidade de São Paulo. Motriz 2007; 13(3): 214-24.

13. Nahas MV. Atividade física, saúde e qualidade de vida. Londrina: Midiograf, 2001. 
Ginástica Laboral: efeitos de um programa de ginástica laboral sobre a flexibilidade em trabalhadores de diversos setores de um hospital de ensino da região do Vale do Rio Pardo/RS

Andréa Mattos de Andrade, Cézane Priscila Reuter, Miriam Beatris Reckziegel, Hildegard Hedwig Pohl, Leandro Tibiriçá Burgos.

14. Brooks D. Treinamento personalizado: elaboração e montagem de programas. São Paulo: Phorte

15. Muyor JM, López-Miñarro PA, Casimiro AJ. Effect of stretching program in an industrial workplace on hamstring flexibility and sagittal spinal posture of adult women workers: a randomized controlled trial. J Voltar Musculoskelet Rehabil 2012; 25(3): 161-169. DOI: http://dx.doi.org/10.3233/BMR2012-0323.

16. REIS, Daiane Villanova. Ginástica Laboral e flexibilidade: um estudo na Secretaria Municipal de Educação de Taquari - RS. 2005. 25f Monografia (Graduação) - Universidade de Santa Cruz do Sul, 2005.
17. Zatarin V., Botolazzo GL. Efeitos da manipulação na articulação sacro-ilíaca e transição lombossacral sobre a flexibilidade da cadeia muscular posterior. Ter Man 2012; 10(47): 40-45. DOI: http:// dx.doi.org/10.9736/TerMan.v10.n47.63.

18. Achour Jr A. Bases para exercícios de alongamento: relacionado com a saúde e no desempenho atlético. Londrina, 1996.

19. Silveira MG, Teixeira MVO, Durães GM, Maia MFM, Lafetá JC. Nível de flexibilidade em praticantes e não praticantes de um programa de ginástica laboral em uma empresa de saneamento básico 2015; 20(205). 